\title{
Preparation and Application of Conductive Textile Coatings Filled with Honeycomb Structured Carbon Nanotubes
}

\author{
Filip Govaert and Myriam Vanneste \\ Centexbel, Belgian Textile Research Centre, Technologiepark 7, 9052 Gent, Belgium \\ Correspondence should be addressed to Filip Govaert; fgo@centexbel.be
}

Received 28 February 2014; Revised 6 June 2014; Accepted 7 June 2014; Published 18 June 2014

Academic Editor: Jun Yang

Copyright ( 2014 F. Govaert and M. Vanneste. This is an open access article distributed under the Creative Commons Attribution License, which permits unrestricted use, distribution, and reproduction in any medium, provided the original work is properly cited.

\begin{abstract}
Electrical conductive textile coatings with variable amounts of carbon nanotubes (CNTs) are presented. Formulations of textile coatings were prepared with up to $15 \mathrm{wt} \%$ of CNT, based on the solid weight of the binder. The binders are water based polyacrylate dispersions. The CNTs were mixed into the binder dispersion starting from a commercially available aqueous CNT dispersion that is compatible with the binder dispersion. Coating formulations with variable CNT concentrations were applied on polyester and cotton woven and knitted fabrics by different textile coating techniques: direct coating, transfer coating, and screen printing. The coatings showed increasing electrical conductivity with increasing CNT concentration. The coatings can be regarded to be electrically conductive (sheet resistivity $<10^{3} \mathrm{Ohm} / \mathrm{sq}$ ) starting at $3 \mathrm{wt} \% \mathrm{CNT}$. The degree of dispersion of the carbon nanotubes particles inside the coating was visualized by scanning electron microscopy. The CNT particles form honeycomb structured networks in the coatings, proving a high degree of dispersion. This honeycomb structure of CNT particles is forming a conductive network in the coating leading to low resistivity values.
\end{abstract}

\section{Introduction}

Textile materials with integrated electrical conductivity make it possible to create intelligent articles with wide ranging applications in sports and work wear, healthcare, and for technical applications. Nowadays, electrically conductive textile applications are made by weaving, knitting, or attaching metal wires in the textile material [1-3]. The homogeneous distribution of the electric current over the total textile surface is pour and only located at the position of the metal wire. In case of electric connectors this specific location of the electric current is desired, but in devices for sensors or electric heating, this concentration of electric current is a source of concern. These high currents can lead to material defects. To avoid this, many wires are introduced to spread the current equally over the textile surface. Textile articles are subjected to processes and activities during their lifetime of use, that is, washing (tumble), drying, and flexing and exposed to varying (outdoor) conditions: humidity, extreme temperatures, irradiation by UV, rain, and so forth. When metal wires and devices are present in this textile, very severe protection is necessary to avoid rapid degradation and corrosion of these materials. This protection is achieved by using insulation cabling, layered structures, and more stiff and thick materials to limit bending and stretching [4-6].

Conductive coatings or finishes for textiles are used in application such as conductive mattress covers for surgical tables or fabrics with shielding for electromagnetic radiation for military applications or work wear. In that case high loading of carbon black is needed to provide sufficient electrical conductivity, while hampering the final textile properties [7].

The integration of carbon nanotubes (CNTs) in coatings for textiles offer good perspectives to obtain electrically conductive textile materials without losing the basic properties of a textile, that is, light weight, stretchable and flexible, comfortable, and ease of use. Conductive textiles by incorporating $\mathrm{CNT}$ through a coating or dyeing process are already discussed [8-10]. This work assesses the usability of CNT in various textile materials and applications by investigating CNT as an additive in coatings for textiles. In this work, textile coatings with variable amounts of CNT were developed and applied by traditional textile application 


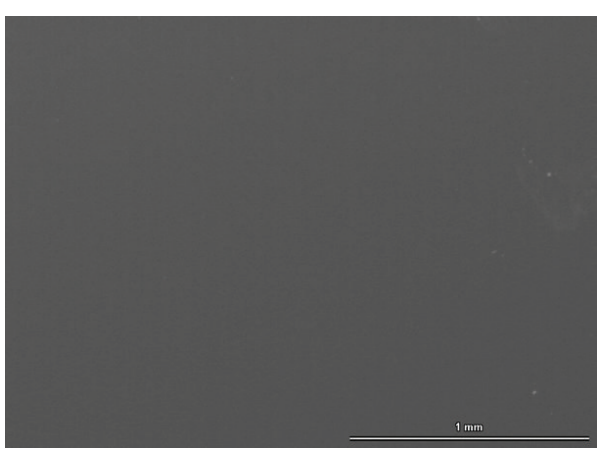

(a)

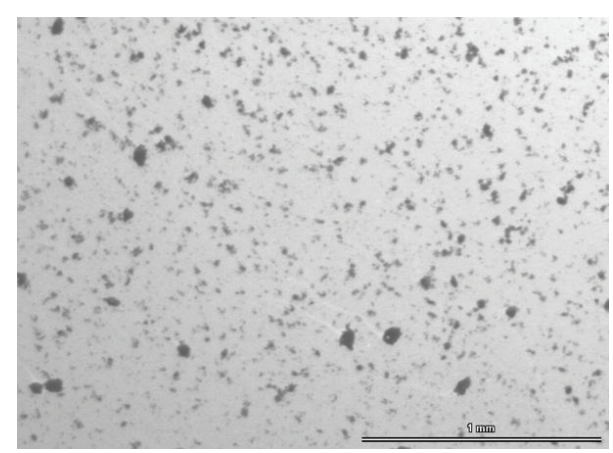

(b)

FIgURE 1: Microscopic evaluation of wet $1 \mathrm{wt} \% \mathrm{CNT}$ coating formulations, with no CNTs visible (a) and agglomerates of CNTs (b).

processes, that is, direct coating, transfer coating, and screen printing. A high degree of dispersion of the CNT within the coating was observed over the entire concentration range and visualized by scanning electron microscopy. This study reveals the construction of a honeycomb conductive network of the CNT particles inside the coatings.

\section{Materials and Methods}

2.1. Materials. The CNTs were supplied by Nanocyl (Belgium) as an aqueous dispersion of $3 \%$ by weight. This dispersion is commercially available under the name of Aquacyl.

As binders, polyacrylate dispersions under the commercial name Lurapret from BASF (Germany) were used. Lurapret dispersions are used to make coatings for home and technical textiles and characterized by a good wash and chemical resistance and a long lifetime. For textile coating, thickeners are added to the formulation, prior to coating, to avoid complete penetration inside the textile material. An acrylate-based polymer synthetic thickener form BASF was used: Lutexal HIT. Furthermore pigment stabilizing agents based on alkoxylated surfactants were used to improve the dispersion of the CNT particles in the formulation.

The textile materials used in this study are polyester knitted and woven fabrics from various suppliers.

For transfer coating process, release paper from ArjoWiggins, UK (Primacast Mirror) was used.

2.2. Coating Formulation. Coating formulations were prepared by mixing different amounts of the CNT dispersion and binder dispersion with a laboratory overhead stirrer (IKA, Germany). The formulating was divided into two parts. Part one is the addition of the CNT dispersion to the binder dispersion. The CNT dispersion was added in small amounts and under constant mixing at slow speed $(\sim 1000 \mathrm{rpm})$. To avoid high thickening of the formulation paste, small amounts of water are added as well. In Part two, the dispersion was mixed at high speed ( 10000 rpm) to obtain homogeneous dispersed CNT coating formulation. Finally, the vessel was placed in a desiccator and subjected to a vacuum process for $30 \mathrm{~min}$ with a maximum vacuum of -1 bar. This was done to avoid the introduction of air bubbles in the coating formulation and afterwards in the coating on the fabric. The quality of the dispersion was controlled by optical microscopy. When agglomerates of CNT particles are observed, the dispersion was qualified as insufficient and not further used. In case a homogeneous black picture of the dispersion was observed, the dispersion was qualified as good. In Figure 1, images of these microscopic observations of both types of dispersions are showed.

2.3. Coating Application. The coating formulation was prepared for application by adding thickener to adjust the viscosity of the coating paste. The thickener was added in small amounts and by slow mixing ( $500 \mathrm{rpm})$. Afterwards, the coating paste was subjected again to a vacuum process for 30 min with a maximum vacuum of -1 bar.

For the application of the coatings, a labcoater LTE$S$ from Mathis (Switzerland) was used. This labcoater is a combination of an applicator and a drying unit.

For the direct coating applications, the coatings were applied in a three-layered structure. First, a basecoat was applied with the technique of knife-in-air. In this way, a thin layer is applied that covers all pores and holes of the fabric. The coating was immediately dried during $3 \mathrm{~min}$ at $150^{\circ} \mathrm{C}$. Next, two layers were applied by the technique of knife-on-roll. The knife gap was set at $200 \mu \mathrm{m}$. After application the coating is dried in two steps: first during $2 \mathrm{~min}$ at $80^{\circ} \mathrm{C}$ and next during $2 \mathrm{~min}$ at $150^{\circ} \mathrm{C}$. This was done to have gradual evaporation of water and to avoid the formation of air bubbles in the coating. Coated fabrics of $300 \times 400 \mathrm{~mm}$ were obtained after the application and curing processes. Several samples were prepared for conductivity measurements, characterization, and further testing.

For the transfer coating applications, two layers of a CNT based coating were applied on transfer paper and subsequently transferred to the textile material with a tiecoat. This tie-coat is based on a polyurethane dispersion. Drying is done in the same two-step procedure as described above for the direct coatings.

Finally the method of screen printing of CNT based formulations was evaluated as well. For this purpose a screen with the design of an electronic circuit was used.

2.4. Microscopic Characterization. Morphology analysis of surface and layer structure was carried out by means of an 


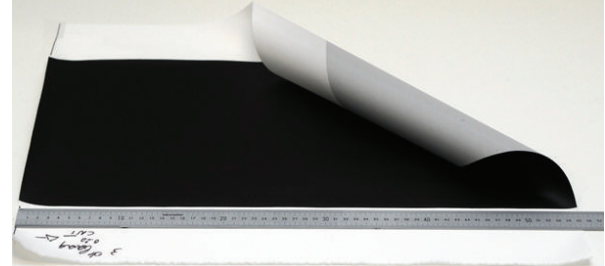

FIGURE 2: Black acrylate-based coating containing $10 \mathrm{wt} \% \mathrm{CNT}$ on a white polyester fabric.

optical microscope, LV100POL (Nikon, Japan) equipped with digital imaging camera and software, DS-Fil (Nikon, Japan).

Scanning electron microscopy (SEM) was used to visualize the CNT particles in the coatings and to evaluate the degree of dispersion of CNT. Two ultrahigh resolution field emission scanning electron microscopes were used, Jeol JSM7600F and Jeol JSM-7500F (Jeol, Japan) in collaboration with Nanocyl.

\section{Results and Discussion}

3.1. Coating Application. The first method used for the application of the CNT containing coating formulations is the direct knife-on-roll coating process. In this process the coating paste passes through a gap between the textile substrate and a knife. This gap determines the amount or weight of coating that is applied onto the textile surface. The 15 different coating formulations containing $1 \mathrm{wt} \%$ of CNT up to $15 \mathrm{wt} \%$ were applied by this process in a 3-layered structure as explained in Section 2. The coatings for evaluating the sheet resistance were prepared by this direct knife coating procedure. A picture of a 3-layered coating system produced on roll-to-roll coating equipment is shown in Figure 2.

The binders used for this type of coatings are high quality polyacrylates for textile coating with good film forming properties, high adhesion, good flexibility, and scratch resistance. These properties are reflected in the coatings containing CNT. Microscopic investigations using an optical microscope equipped with digital imaging are shown in Figure 3. It is clearly seen on these $10 \mathrm{wt} \%$ samples that a homogeneous coating is obtained that is free of defects. Depending on the wt $\%$ of CNT in the coating, differences in surface morphology are observed. At low CNT concentration, the coating has a semigloss aspect. This is similar to the coating without CNT. From CNT amounts of $10 \mathrm{wt} \%$ and higher the coating has a more matt aspect, due to the high solid content or the low amount of binder present in such coatings.

The irregularities observed on the surface can be explained by the roughness of the textile substrate. This can be seen on the image of the cross section where the waviness of the coating is following the structure of the woven fabric. Depending on the type of application method (direct coating versus transfer coating) the coating morphology on the textile can be changed. By the method of transfer coating, the structure of the release paper will determine the surface structure of the top layer. In Figure 4, the cross section of a transfer coated layer system is depicted. As transfer paper, a high-gloss (mirror) type of paper was used. In this process, two layers are subsequently applied on the glossy release paper and fully dried and cured. To transfer this coating system to a textile, a tie-coat (binder without CNT) is applied on this coating system and a piece of textile is rolled in this wet layer. After drying and curing, the textile together with the complete coating system can be released from the paper. With this application process, a smooth and flat coated surface is achieved, due to the use of a mirror type of release paper.

A third application that was tested in this study is the method of screen printing. For this purpose simple screen designs were used to evaluate the printing process. Initially, the same formulation and preparation method was used as for the direct coating application method. The formulation with $10 \mathrm{wt} \% \mathrm{CNT}$ was prepared and applied. Initially no sharp design of the print was obtained. This is due to a slight flow of the formulation immediately after application. This flow is beneficial in knife coating application to obtain a smooth homogeneous film, but in printing application, this flow is not acceptable. The formulation was adapted for screen printing by adding a thixotropic thickener to the formulation. By adding 0.5 to $1 \%$ of Lutexal TX4744 (BASF) to the total formulation, prints with sharp borders and edges were obtained. Examples of such prints are depicted in Figure 5. With this method conductive tracks can be applied on textile fabrics. The resistivity of these tracks depends on the width and thickness of the printed lines.

3.2. Sheet Resistance. In order to assess the influence of the CNT concentration in the coating on the electrical conductivity, 15 coating formulations were prepared with different concentrations of CNT and the sheet resistance of dried and cured coatings measured. The 15 coating formulations consist of $1 \mathrm{wt} \%$ up to $15 \mathrm{wt} \%$ of CNT based on the solid weight of the binder. These formulations were used to apply a three-layered coating system on the fabric by the direct coating process (one layer knife-in-air, two layers knife-onroll). The sheet resistance was measured directly on the coated samples with a four-probe sheet resistance measurement. The results obtained for an acrylic-based textile coating are shown in Figure 6. There is a direct relationship between the concentration of CNT in the coating and the sheet resistance as expected: increasing the amount of CNT in the coating decreases the sheet resistance. A sheet resistance down to $60 \Omega$ /sq was measured at $10 \mathrm{wt} \% \mathrm{CNT}$.

As depicted in Figure 6, it can be seen that percolation starts between 3 and $4 \mathrm{wt} \%$. The coatings can be regarded to be electrically conductive (sheet resistivity $<10^{3} \mathrm{Ohm} / \mathrm{sq}$ ) starting at $3 \mathrm{wt} \% \mathrm{CNT}$. For industrial use, it is important to see that a broad range of electrical conductivity levels can be obtained by simply changing the amount of CNT in the coating. Above a concentration of $10 \mathrm{wt} \% \mathrm{CNT}$, no significant decrease of sheet resistance is measured.

3.3. Dispersion of Carbon Nanotubes. By a controlled preparation of CNT based formulations-slow addition of the CNT dispersion, counter the effect of viscosity increase by the addition of small amounts of water-a high degree of dispersion of the CNT in the binder matrix can be obtained. Mixtures of alkoxylates improve strongly the rheology of the formulation. 


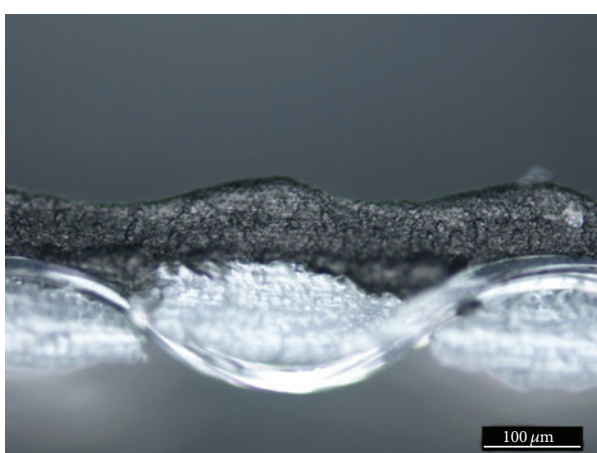

(a)

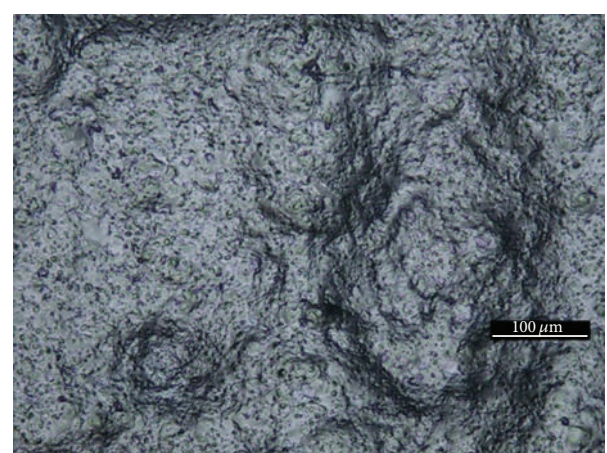

(b)

Figure 3: Microscopic images of coated textiles with a black acrylate-based coating containing 10 wt\% CNT on a white polyester fabric: a cross section (a) and the surface (b).

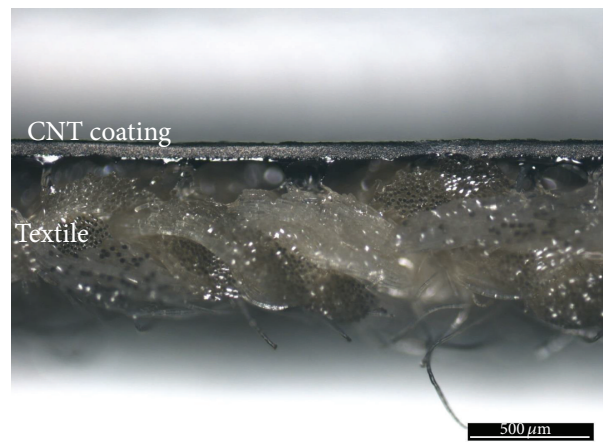

FIGURE 4: Microscopic image (cross section) of a coated textile with a black acrylate-based coating containing $10 \mathrm{wt} \%$ CNT on a polyester textile fabric by the transfer coating process.

Moreover by adding these alkoxylated surfactants to the formulation, the CNTs orientate during the coating drying process in a way that they form a honeycomb structure inside the binder matrix. This structure can be visualized by scanning electron microscopy images of applied coatings, depicted in Figure 7. The electrically conductive CNTs are highlighted during imaging and a structured network is visible.

This structure forms a continuous network inside the coating at a concentration of $10 \mathrm{wt} \%$ of CNT, based on the solid content. At lower CNT content, this network is inside the coating as well, but not homogeneously present over de complete surface. This is shown in Figure 8(a) for a coating with a CNT content of $6 \mathrm{wt} \%$. The absence of a complete network results in a higher sheet resistance. At a concentration above $10 \mathrm{wt} \%$ of CNT in the coating, this network is in some areas overloaded with CNT, as shown in Figure 8(b) for a coating with a CNT content of $13 \mathrm{wt} \%$.

The SEM analysis shows that a high degree of dispersion of carbon nanotubes can be observed. A network structure appears together with a high value of conductivity. From 3 wt $\%$ CNT the first network structure appears. This is also the concentration where a more linear relation between concentration and resistivity starts (see Figure 6). The most clear network structure can be observed at 10 and $11 \mathrm{wt} \%$. Above this concentration saturation of carbon nanotubes in some areas occurs and at $15 \%$ loss of structure is observed. The conductivity increases slightly from $4 \mathrm{wt} \%$ up to $14 \mathrm{wt} \%$ and drops slightly at $15 \mathrm{wt} \%$.

The origin of this network can be explained by the fact that the CNT particles are homogeneously present in the acrylate binder dispersion. During the drying process of the wet coating, all the water evaporates and the acrylate particles move together surrounded by the CNT particles. The CNT particles settle between the acrylate particles forming a honeycomb structured network. In the SEM picture in Figure 9 one can observe the surface of an acrylate-based coating. This coating was completely dried at $30^{\circ} \mathrm{C}$ to avoid fusion of the acrylate particles at high temperature. In this picture the individual acrylate particles can be observed. The particles have an average particles size of approximately $180 \mathrm{~nm}$. This size corresponds to honeycomb cell size that can be observed in Figure 7. Measurements show that these CNT cells have sizes between 160 and $230 \mathrm{~nm}$. The most perfect CNT honeycomb cells have a diameter of approximately $180 \mathrm{~nm}$.

\section{Conclusions}

This work describes the introduction of electrical conductivity in textile materials by the use of textile coatings filled with carbon nanotubes. The preparation of coating 


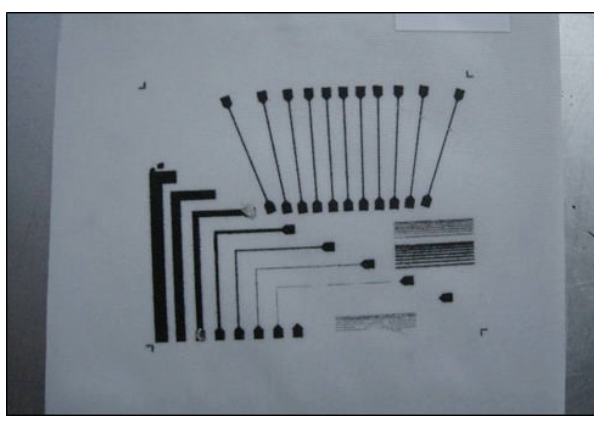

(a)

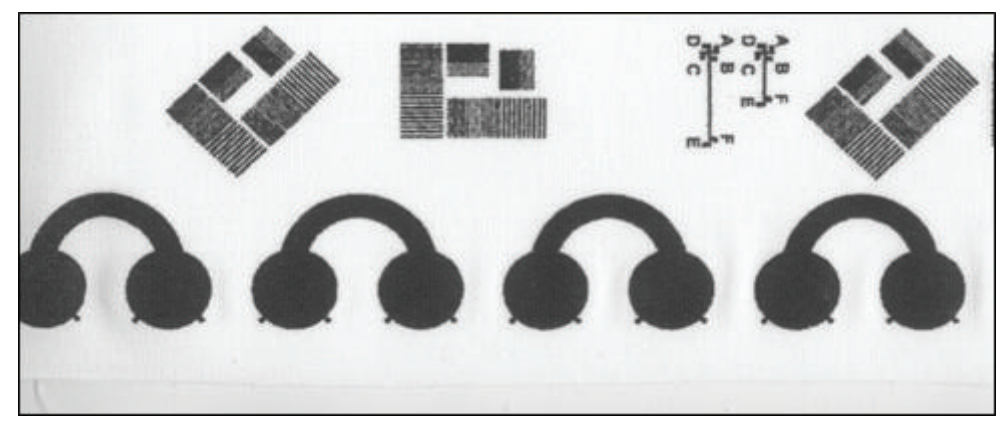

(b)

FIGURE 5: Screen printing of black acrylate-based coatings containing $10 \mathrm{wt} \% \mathrm{CNT}$ on polyester (a) and cotton (b) textile fabric.

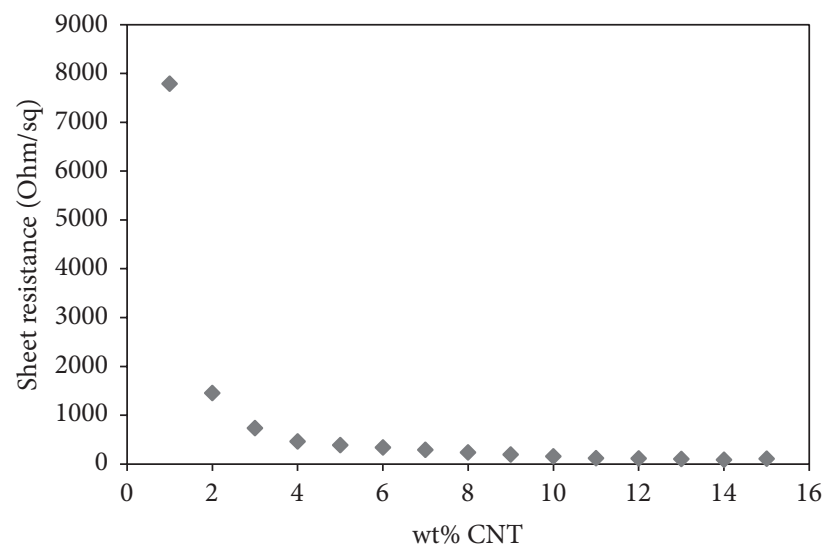

FIGURE 6: Sheet resistance of an acrylic-based textile coatings with different CNT loading (wt\%).

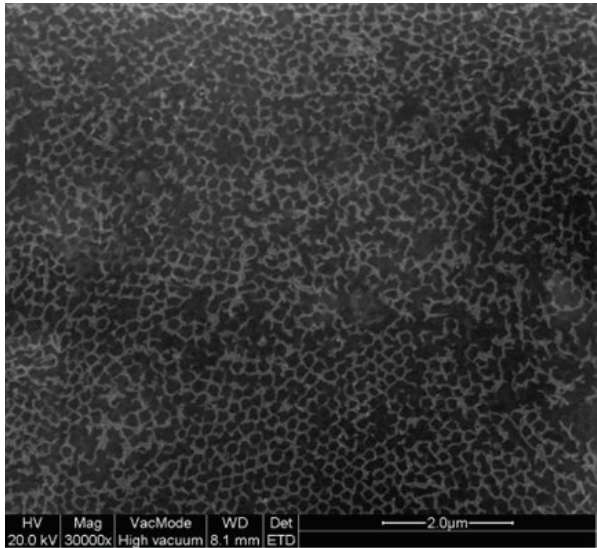

(a)

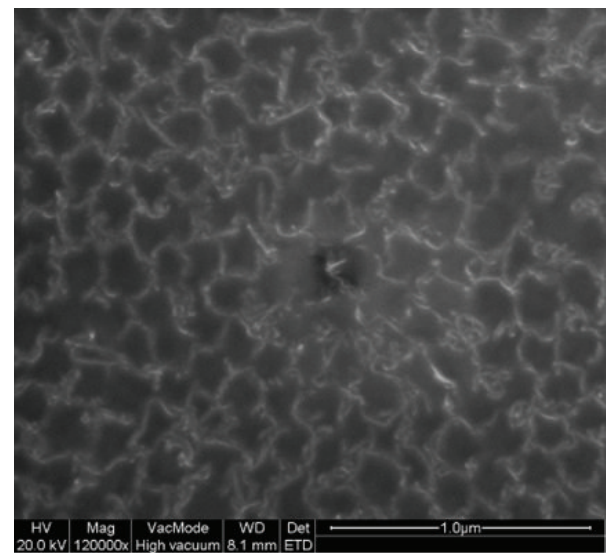

(b)

FIGURE 7: Scanning electron microscopy images of the surface of an acrylate-based coating containing $10 \mathrm{wt} \%$ CNT, in low (a) and high (b) magnification.

formulations of water based acrylic binders with CNT as the active filler material is demonstrated. It is important to control the viscosity of the formulation during the addition of the CNT. CNT acts as a thickener to the binder material during the preparation of the coating paste. This viscosity increase must be controlled by adding CNT in small amounts to the binder material and by adding water to counter the thickening effect. The application of these coating formulations was demonstrated by direct coating, transfer coating, and screen printing. With the direct and transfer coating process, full coatings of one or more layers are applied over the complete textile surface. In direct coating, the textile structure is maintained, but with the transfer coating process, flat and smooth coating surfaces can be obtained onto a textile substrate. With screen printing, conductive tracks or designs are applied on the textile material. The level of conductivity of these layers or tracks can be defined by the coating composition. By varying the amount of CNT in 


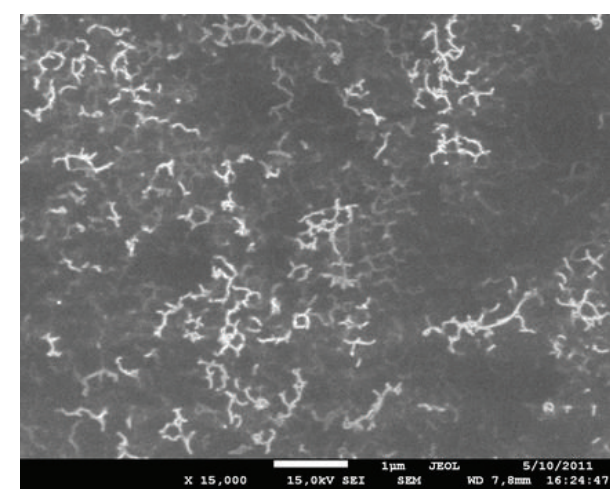

(a)

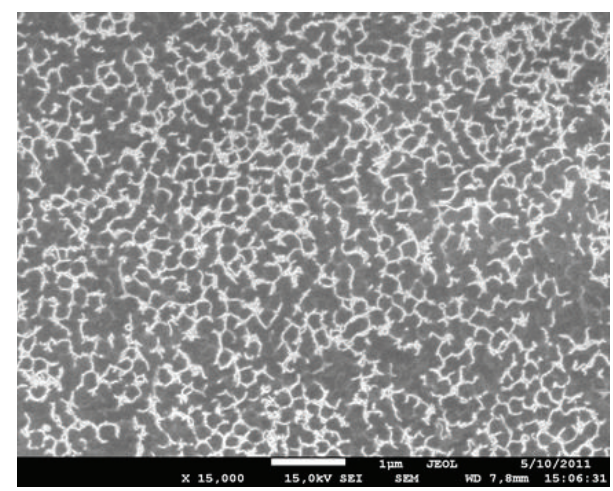

(b)

FIGURE 8: SEM images of the surface of an acrylate-based coating containing 6 wt $\%$ CNT (a) and 13 wt $\%$ CNT.

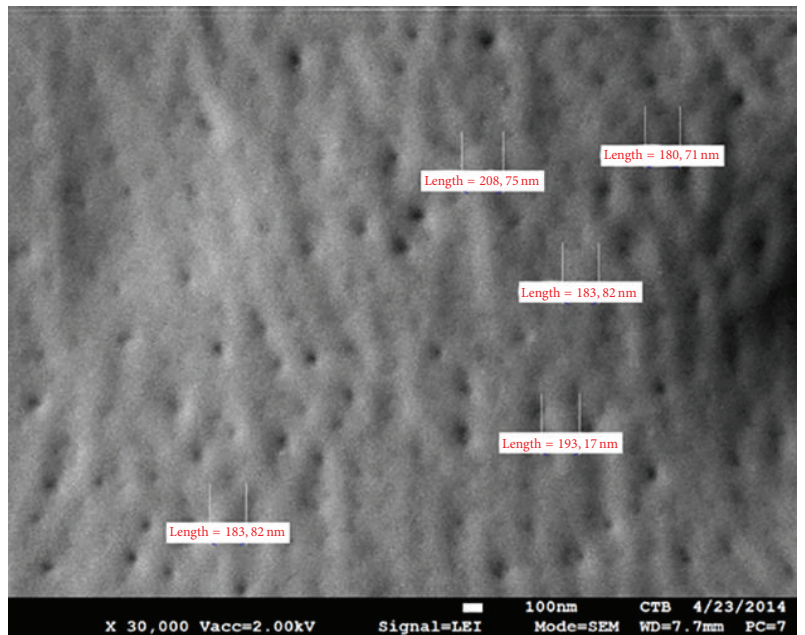

FIGURE 9: SEM image from the surface of an acrylate-based coating dried at $30^{\circ} \mathrm{C}$ with indication of the acrylate particle size.

the coating formulation, the range of conductivity can be tuned from antistatic ( $1 \mathrm{wt} \% \mathrm{CNT}$ ) up to high conductivity (10 wt\% CNT). The high level of conductivity (low resistivity) was explained by a high degree of dispersion of the CNT particles in the coating. A network of honeycomb structured CNTs can be visualized by scanning electron microscopy. This structure is homogeneously present in the coating at a concentration from $10 \mathrm{wt} \%$ to $11 \mathrm{wt} \%$ of CNT. The CNT network can be explained by the fact that the CNT particles are homogeneously present in the acrylate binder dispersion. During the drying process of the coating formulation, the acrylate particles move together due to the evaporation of the water. The CNT particles are moving between the acrylate particles forming a honeycomb structured network. The cell size of this CNT network corresponds to the size of the acrylate particles.

\section{Conflict of Interests}

The authors declare that there is no conflict of interests regarding the publication of this paper.

\section{Acknowledgments}

The research results presented in this paper have been obtained in the research project IWT-095070 and IWT120629, with the financial support of the Flemish government agency for Innovation by Science and Technology. The authors are grateful to Nanocyl for providing the CNT material and for the fruitful discussions, SEM measurements, and collaboration.

\section{References}

[1] R. F. Service, "Electronic textiles charge ahead," Science, vol. 301, no. 5635, pp. 909-911, 2003.

[2] M. Catrysse, R. Puers, C. Hertleer, L. Van Langenhove, H. Van Egmond, and D. Matthys, "Towards the integration of textile sensors in a wireless monitoring suit," Sensors and Actuators A: Physical, vol. 114, no. 2-3, pp. 302-311, 2004.

[3] I. Locher, T. Kirstein, and G. Tröster, "Temperature profile estimation with smart textiles," in Proceedings of the 1st International Scientific Conference (Ambience '05), Tampere, Finland, September 2005.

[4] E. R. Post, M. Orth, R. R. Russo, and N. Gershenfeld, "E-broîdery: design and fabrication of textile-based computing," IBM Systems Journal, vol. 39, no. 3-4, pp. 840-860, 2000.

[5] S. Coyle, K. -T. Lau, N. Moyna et al., "BIOTEX-biosensing textiles for personalised healthcare management," IEEE Transactions on Information Technology in Biomedicine, vol. 14, no. 2, pp. 364-370, 2010.

[6] M. Billinghurst, "New ways to manage information," Computer, vol. 32, no. 1, pp. 57-64, 1999.

[7] D. Negru, C.-T. Buda, and D. Avram, "Electrical conductivity of woven fabrics coated with carbon black particles," Fibres and Textiles in Eastern Europe, vol. 90, no. 1, pp. 53-56, 2012.

[8] M. I. H. Panhuis, J. Wu, S. A. Ashraf, and G. G. Wallace, "Conducting textiles from single-walled carbon nanotubes," Synthetic Metals, vol. 157, no. 8-9, pp. 358-362, 2007.

[9] B. S. Shim, W. Chen, C. Doty, C. Xu, and N. A. Kotov, "Smart electronic yarns and wearable fabrics for human biomonitoring made by carbon nanotube coating with polyelectrolytes," Nano Letters, vol. 8, no. 12, pp. 4151-4157, 2008.

[10] R. Zhang, H. Deng, R. Valenca et al., "Carbon nanotube polymer coatings for textile yarns with good strain sensing capability," Sensors and Actuators A: Physical, vol. 179, pp. 83-91, 2012. 

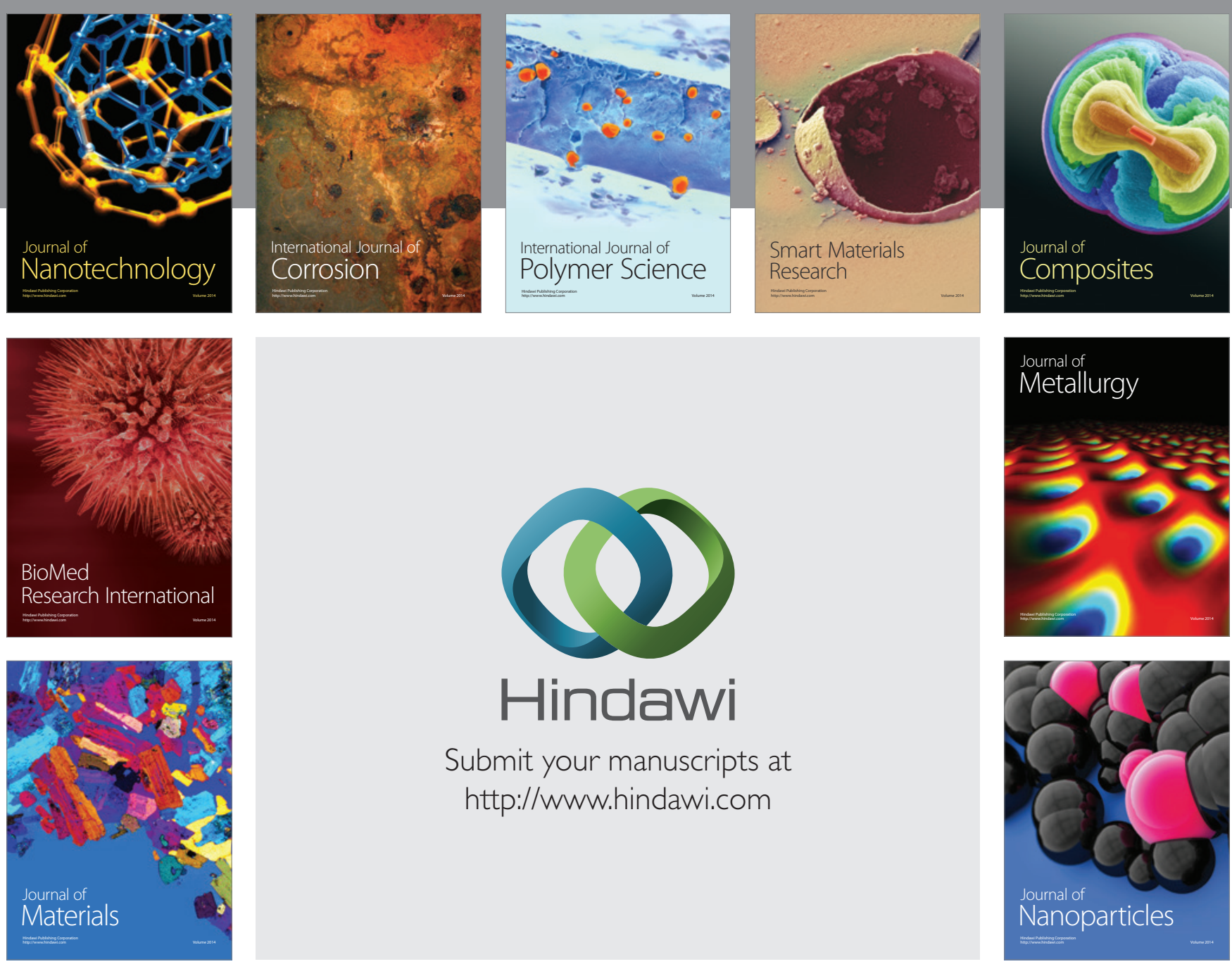

Submit your manuscripts at http://www.hindawi.com
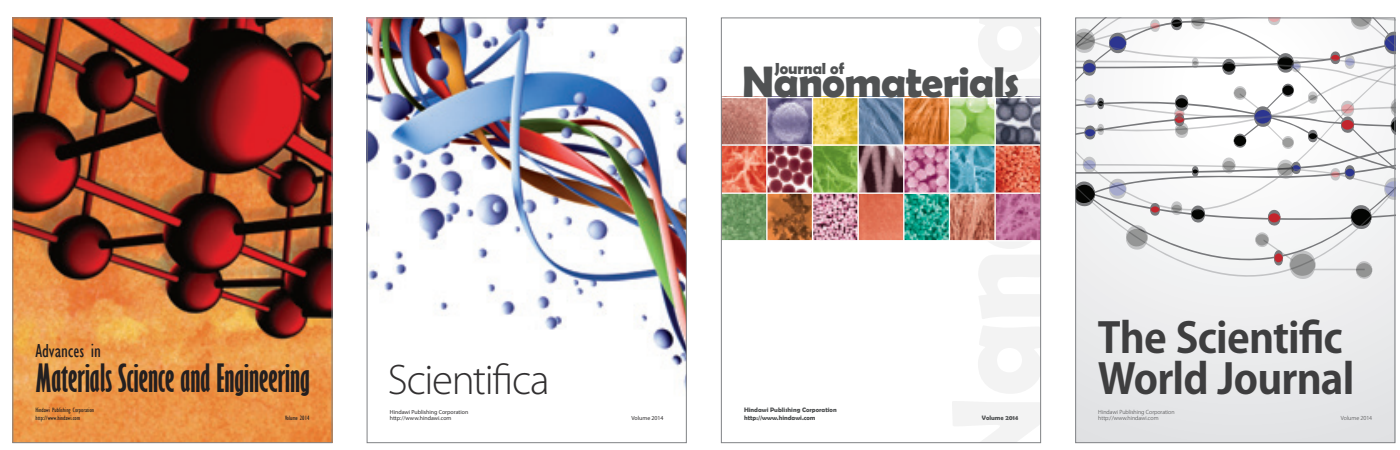

\section{The Scientific World Journal}
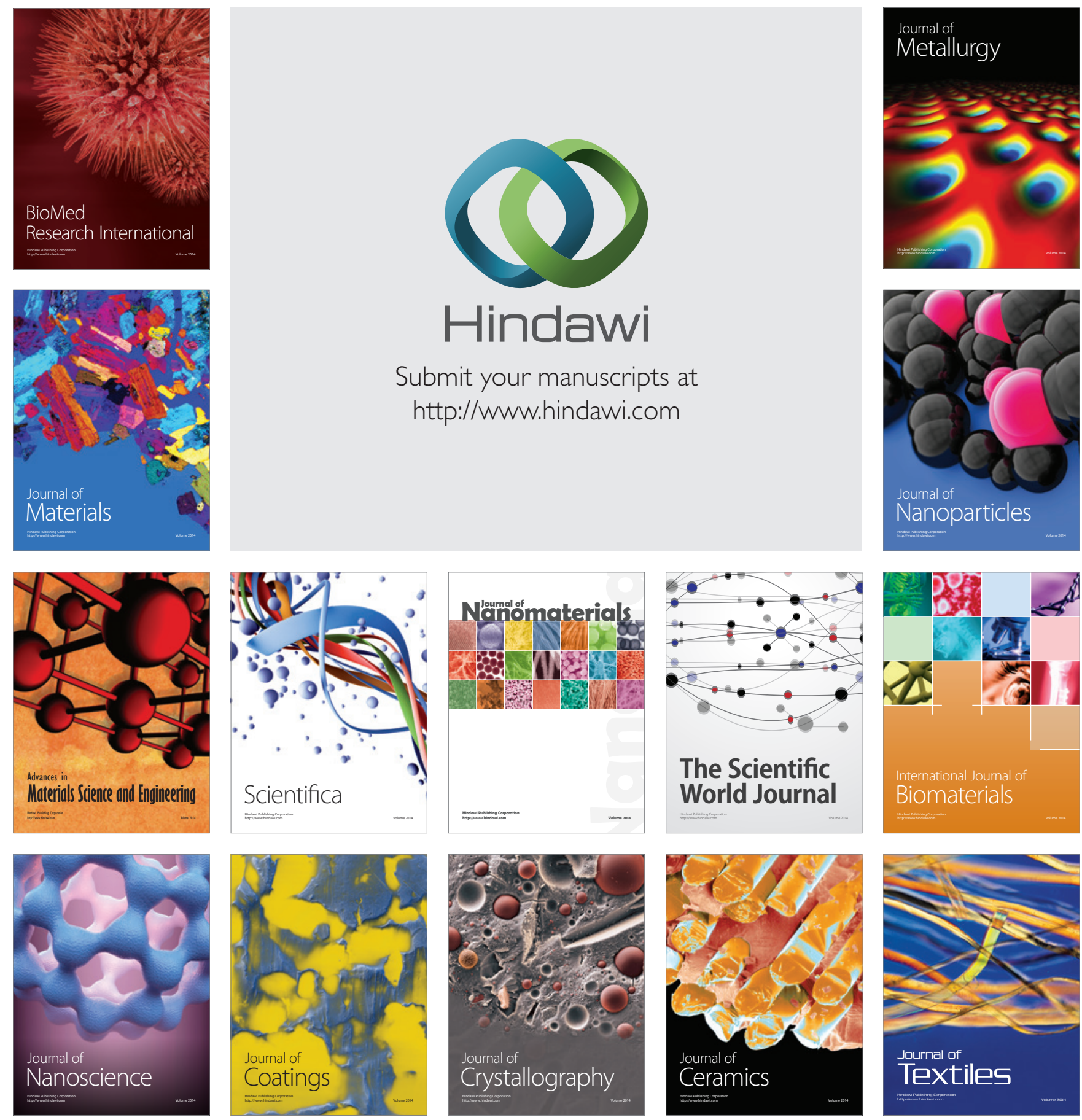